\title{
Analysis of Commutation Failure in Multi-Infeed HVDC System under Different Load Models
}

\author{
Chengjun Xia1, Zhongchao Yang1, Kun Men², Yong Zhao \\ ${ }^{1}$ School of Electric Power, South China University of Technology, Guangzhou, China \\ ${ }^{2}$ Electric Power Research Institute of CSG, China Southern Power Grid, Guangzhou, China \\ Email: y zhongchao@163.com
}

Received June 2014

\begin{abstract}
HVDC technology has been widely used in modern power system. On one hand, HVDC has the advantages of economy, high efficiency and strong controllability. While on the other hand, it makes the dynamic characteristics of the power system becoming more and more complex. That puts forward a new challenge to system stability and raises new questions for power system simulation. This paper focuses on the interaction between AC and DC systems, especially the problem of commutation failure caused by AC system fault. Based on the data of China Southern Power Grid, this paper calculates the fault regions that may cause commutation failure and calculates the system critical clearance time under different load models, analyzes the impacts of different load models on commutation failure and the stability of AC/DC hybrid system.
\end{abstract}

\section{Keywords}

AC/DC Hybrid System, Commutation Failure, Voltage of Converter Bus, Load Model, Power System Simulation

\section{Introduction}

The HVDC transmission system has the advantages of high capacity, high efficiency and strong controllability, and it had been widely used in power system [1]. As the development of power electronics and control technology, the HVDC will have a broad application in the future. However, the HVDC also puts forward some new challenges to system operation, the AC/DC interaction has become an emphasis for researchers [2] [3].

In all sorts of problems exposed in AC/DC hybrid system, commutation failure caused by AC system fault is the most common one. Commutation failure can lead to DC voltage sag, DC current swell, DC power decrease, reduction of converter valve service life, DC magnetic biasing of converter transformer and overvoltage of "weak" receiving end system. For multi-infeed HVDC system, the electrical distance between each inverter station is short, the AC/DC interaction features are more complex, commutation failure occurs in one inverter station may lead to commutation failure of nearby inverter stations at the same time. In severe cases, that may lead to DC power interruption, which will be a serious threat to the stability of AC/DC hybrid system [4]-[6]. 
Research on commutation failure is significant for understanding the operation features of AC/DC hybrid system, improving the system operating condition and enhancing the system stability. Researchers had carried out large amount of research work. Reference [7] [8] focused on the extinction angle $\gamma$, analyzed the factors of commutation failure in detail. Reference [9] pointed out that the voltage sag of converter bus caused by AC system fault is the main cause of commutation failure. In [10] and [11], a new index-CFII (Commutation Failure Immunity Index) was proposed to evaluate the converter's resistance to commutation failure cause by AC system fault. In [12], a criterion of commutation failure was proposed based on a great deal of simulation study. However, the previous research paid less attention to the load model. In fact, it can be found from the simulation result that, the occurrence of HVDC commutation failure has a close relationship with load model. This paper points out that the mechanical inertia of the motor load will affect the occurrence of commutation failure. Simulation is carried out to verify the viewpoint proposed in this paper.

\section{Mechanism and Factors of Commutation Failure}

The occurrence of commutation failure caused by AC system fault is related to the strength of receiving end system. The "stronger" the receiving end is, the weaker the AC/DC influence each other and the less probable the commutation failure will occur. The strength of receiving end system can be evaluated from two aspects.

On one hand, the strength can be evaluated by the indicator SCR (Short Circuit Ratio), which is defined as follow

$$
\mathrm{SCR}=\frac{\mathrm{S}}{P_{d N}}
$$

where $S$ is the three-phase short-circuit capacity of receiving end system when the voltage of converter bus is at 1 p.u. $P_{d N}$ is the rated power of HVDC. In [13] the strength of AC system was divided into three levels according to SCR: high (SCR $>3$ ), low $(2 \leq \mathrm{SCR} \leq 3)$, very low $(\mathrm{SCR}<2)$.

On the other hand, the system strength can be evaluated by the mechanical inertia. If the mechanical inertia is large enough, the potential of generators will maintain when fault occurs, that will be beneficial for voltage recovery and preventing system from collapsing. It can be said that the larger the mechanical inertia is the stronger the receiving end system will be. Generator rotors are the main source of mechanical inertia, but it should be pointed out that, except from generators, the motor load can also provide mechanical inertia.

The thyristors used in HVDC system is a kind of half controlled component, the commutation process needs a certain time for internal carrier recombination and blocking ability restoring. The time of carrier recombination process can be evaluated by extinction angle $\gamma \cdot \gamma$ is important for the operation of inverter, if the operating $\gamma<$ $\gamma_{\min }$, the internal carrier of thyristors will not have enough time to recombine and commutation failure will occur. Extinction angle $\gamma$ can be described as follow [14]

$$
\gamma=\arccos \left(\frac{\sqrt{2} k I_{d} X_{c}}{E}+\cos \beta\right)
$$

where $E$ is the voltage of converter bus, $k$ is the ratio of converter transformer, $\beta$ is the advance trigger angle, $I_{d}$ is the DC current, $X_{c}$ is the commutation reactor. For a certain HVDC, its commutation reactor could be considered as a constant. When fault occurs in receiving end AC system, the DC controllers and the tap changers cannot response immediately. So the $\beta$ and $k$ remain unchanged at the instance of fault. The increase of DC current or the decline of converter bus voltage will lead to the decrease of $\gamma$. If $\gamma$ decreases below $\gamma_{\text {min }}$, commutation failure will occur. The influence of converter bus voltage on commutation failure is this paper mainly concerns about.

For multi-infeed HVDC system, when short circuit fault occurs in the receiving end, the voltage of converter bus will decline, and that may results in commutation failure in more than one inverter stations at the same time. In power system, there are many kinds of load. Different load models will have different impact on the decline of voltage, thus influence the occurance of commutation failure.

\section{Impact of Different Load Models on Voltage Decline}

\subsection{Static Load Model}

The static load model can reflect the change of active and reactive load as the frequency and voltage change 
slowly [15]. ZIP model is a kind of static load, and it is most widely used in simulation. The ZIP load consists of three components: constant impedance load, constant current load and constant power load. It can be described as follow

$$
\left\{\begin{array}{l}
P_{L}=P_{0}\left[P_{Z}\left(\frac{V}{V_{0}}\right)^{2}+P_{I}\left(\frac{V}{V_{0}}\right)+P_{P}\right]\left(1+\Delta f \cdot L_{P f}\right) \\
Q_{L}=Q_{0}\left[Q_{Z}\left(\frac{V}{V_{0}}\right)^{2}+Q_{I}\left(\frac{V}{V_{0}}\right)+Q_{P}\right]\left(1+\Delta f \cdot L_{Q f}\right)
\end{array}\right.
$$

where $P_{Z}, Q_{Z}$ are the percentage of constant impedance active and reactive load; $P_{I}, Q_{I}$ are the percentage of constant current active and reactive load; $P_{P}, Q_{P}$ are the percentage of constant power active and reactive load, and satisfy $P_{Z}+P_{I}+P_{P}=Q_{Z}+Q_{I}+Q_{P}=1 . P_{0}, Q_{0}, V_{0}$ are active load, reactive load and voltage of the system under steady state. $\Delta f$ is the deviation of the actual value and steady-state value of system frequency.

When fault occurs in AC system, the voltage will decline immediately, and the frequency remains unchanged at the instance of fault. It can be seen from Equation (3) that the constant impedance load is proportional to the square of voltage, so its reactive power consumption will drop rapidly as the voltage decline after fault, which is beneficial for preventing the voltage from declining further. The constant power load is irrelevant to voltage. Its reactive power consumption will not change as the voltage decline and it makes no contribution to preventing voltage decline [16]. The constant current load is proportional to first power of the voltage. Its contribution to preventing voltage sag is between constant impedance load and constant power load.

\subsection{Dynamic Load Model}

In comparison with static load, the dynamic load can reflect the change of active and reactive load relative to the fast change of frequency and voltage. There are many kinds of dynamic load in power system, and the motor load is the most common one. The simple equivalent circuit of motor is showed in Figure 1. Where $\dot{U}$ is the input voltage, $\dot{I}$ is the rotor current, $X_{m}$ is the excitation reactance, $X_{r}$ is the rotor leakage reactance, $R_{r}$ is the rotor resistance, $s$ is the slip. The reactive power consumption of the motor load can be described as follow [17]

$$
Q=Q_{m}+Q_{r}=U^{2} / X_{m}+I^{2} X_{r}
$$

where $Q_{m}=U^{2} / X_{m}$ is the exciting power of the motor, $Q_{r}=I^{2} X_{r}$ is the reactive power consumption of rotor leakage reactance. Variable resistance $R_{r} / s$ in Figure 1 is equivalent to the mechanical load of the motor, and the mechanical load $P$ can be described as follow

$$
P=I^{2} R_{r} / s
$$

From Equation (4) we can see that the exciting power $Q_{m}$ is proportional to the square of voltage, so it will reduce significantly as voltage sags after fault. Because of the inertia of motor rotor, the speed of motor cannot change suddenly, so the slip also remains unchanged at the instance of fault. Assume that the mechanical load of the motor also remains the same. We can see from Equation (5) that the rotor current will maintain the same, thus the reactive power consumption of rotor leakage reactance will not increase. That means the motor load, as revolving equipment, can remain its speed unchanged at the instance of fault because of its inertia, and reduce its exciting power significantly without increasing the reactive power consumption of rotor leakage reactance at the same time. Compared with ZIP load, the motor load is better in enhancing the system voltage support.

However, in the duration of disturbance, as the electromagnetic torque of motor is proportional to the square of the input voltage $U$, the decline of voltage will lead to the reduction of electromagnetic torque. If the mechanical torque of motor load remains unchanged, the speed will drop continuously until the rotor is blocked. In

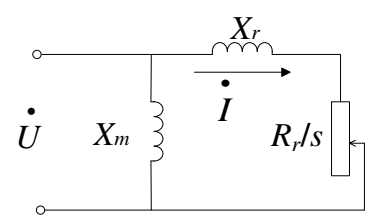

Figure 1. The equivalent circuit of induction motor. 
this process, the slip will increase and approximate to 1. From Equation (5), the current $I$ will increase, so do the reactive power consumption of rotor leakage reactance, and that is not good for voltage recovering [18]-[20].

\section{Simulation}

\subsection{Criterion of Commutation Failure}

The paper adopts the criterion proposed in [12]: when using BPA to do the simulation, set three-phase fault in different position of the system, if the three-phase voltage of converter bus decline below $90 \%$ the value before fault, commutation failure is consider to occur.

\subsection{Simulation of Commutation Failure}

The simulation of this paper is based on the data of China Southern Power Grid (CSG) which is the largest and most complex AC/DC hybrid power system. There will be totally 7 HVDCs in 2014: Tian-guang, Yun-guang, Xin-an, Gao-zhao, Jiangcheng, Nuozhadu and Xiluodu. All these HVDCs terminate at Guangdong. A simple diagram of HVDCs in CSG is show in Figure 2.

The load models of simulation include ZIP load, 40\% motor load and 60\% constant impedance load in parallel, 50\% motor load and 50\% constant impedance load in parallel, 60\% motor load and 40\% constant impedance load in parallel. The ZIP load is consisted of $30 \%$ of constant impedance load, $40 \%$ of constant current load and $30 \%$ of constant power load. The parameters of motor load are showed in Table 1.

Three-phase fault is set to occur at $500 \mathrm{kV}$ nodes in Guangdong. Calculate the bus voltage of inverter stations and use the criterion proposed in [12] to judge the occurrence of commutation failure. A simulation result is showed in Table 2. In this case, the fault is set at $500 \mathrm{kV}$ substation Pengcheng.

From Table 2 we can see that when the load model is motor and constant impedance in parallel, the decline of bus voltage reduce significantly at the instance of fault. As the percentage of motor load increase, decline of bus voltage reduce further.

To make the simulation result more intuitive, Figures 3-6 show the fault region that may result in commutation failure occur in more than three inverters.

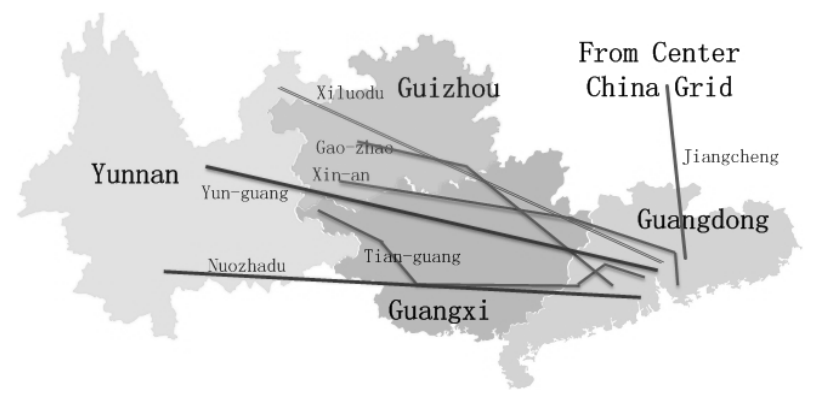

Figure 2. Simple diagram of HVDCs in CSG.

Table 1. The parameter of induction motor model.

\begin{tabular}{cc}
\hline Parameter & Value \\
\hline Inertia time constant (s) & 2 \\
Load rate & 0.51 \\
Stator resistance (p.u.) & 0 \\
Stator reactance (p.u.) & 0.18 \\
excitation reactance (p.u.) & 3.5 \\
Rotor resistance (p.u.) & 0.02 \\
Rotor reactance (p.u.) & 0.12 \\
Constant A of mechanical torque equation & 0.8875 \\
Constant B of mechanical torque equation & -0.04 \\
\hline
\end{tabular}


Table 2. Voltage sag and commutation failure when fault occurs at Pengcheng.

\begin{tabular}{|c|c|c|c|c|c|}
\hline & & ZIP load & $40 \%$ motor load & $50 \%$ motor load & $60 \%$ motor load \\
\hline \multirow{2}{*}{ Xin-an } & Percentage of the voltage before fault (\%) & 4.37 & 6.54 & 6.87 & 7.15 \\
\hline & Commutation failure occurance & Yes & Yes & Yes & Yes \\
\hline \multirow{2}{*}{ Gao-zhao } & Percentage of the voltage before fault (\%) & 92.78 & 97.93 & 98.22 & 98.44 \\
\hline & Commutation failure occurance & No & No & No & No \\
\hline \multirow{2}{*}{ Tian-guang } & Percentage of the voltage before fault (\%) & 86.52 & 96.10 & 96.72 & 97.17 \\
\hline & Commutation failure occurance & Yes & No & No & No \\
\hline \multirow{2}{*}{ Jiangcheng } & Percentage of the voltage before fault (\%) & 79.08 & 88.28 & 89.18 & 89.90 \\
\hline & Commutation failure occurance & Yes & Yes & Yes & Yes \\
\hline \multirow{2}{*}{ Yun-guang } & Percentage of the voltage before fault (\%) & 81.65 & 90.89 & 91.68 & 92.30 \\
\hline & Commutation failure occurance & Yes & No & No & No \\
\hline \multirow{2}{*}{ Nuozhadu } & Percentage of the voltage before fault (\%) & 95.68 & 98.90 & 99.07 & 99.18 \\
\hline & Commutation failure occurance & No & No & No & No \\
\hline \multirow{2}{*}{ Xiluodu } & Percentage of the voltage before fault (\%) & 85.97 & 94.94 & 95.52 & 95.96 \\
\hline & Commutation failure occurance & Yes & No & No & No \\
\hline
\end{tabular}

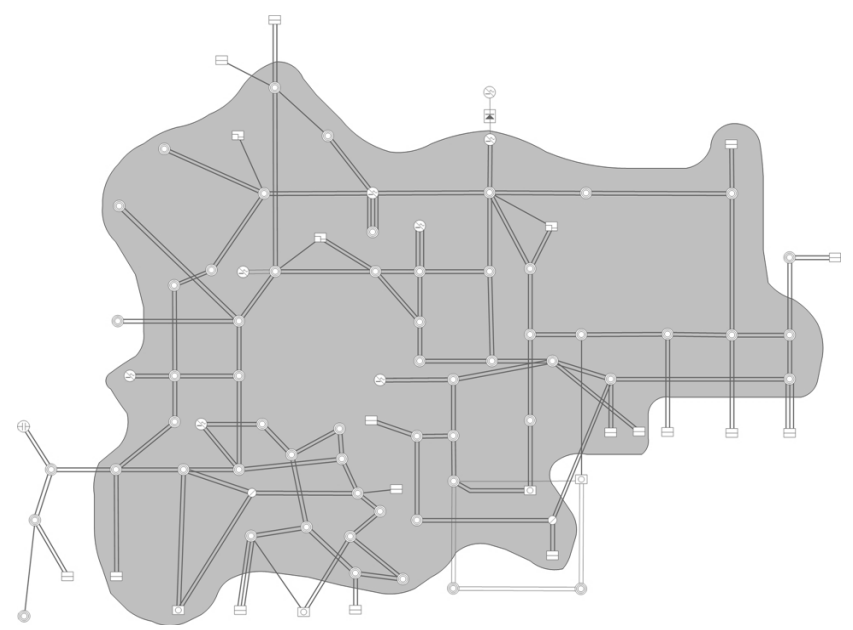

Figure 3. Fault region under ZIP load.

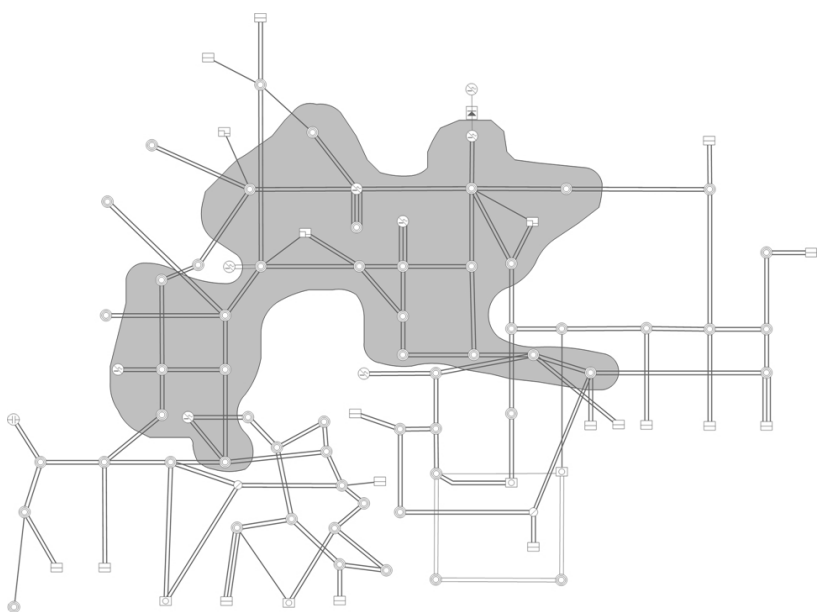

Figure 4. Fault region under $40 \%$ of motor load. 


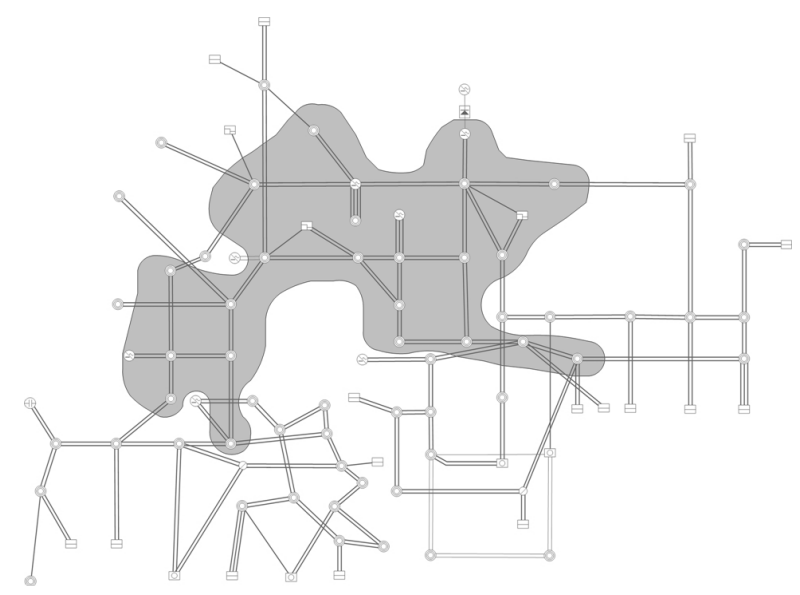

Figure 5. Fault region under 50\% of motor load.

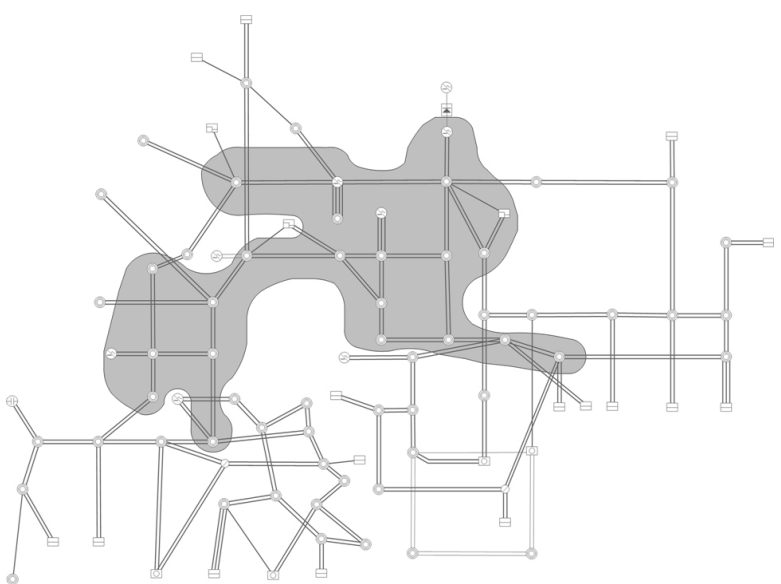

Figure 6. Fault region under $60 \%$ of motor load.

From the four figures above we can see that when using motor load in simulation, the fault region decrease significantly than using ZIP load. As the proportion of motor load increase, the fault region decrease further. That demonstrates the viewpoint of Section 3.2: the motor load can provide inertia to the system, so it is better than ZIP load in supporting the system voltage and reducing the possibility of commutation failure.

Different HVDC show different sensitivity to AC system fault. Table 3 lists the number of fault node that may lead to commutation failure at different inverter. The number of fault nodes can, to a certain extent, show the HVDCs' sensitivity to AC system fault.

From Table 3 we can see that Xiluodu HVDC has the highest sensitivity to AC system fault, while Xin-an HVDC is relatively lower. Overall, the sensitivity of each HVDC decreases as the percentage of motor load increase.

\subsection{Simulation of System Transient Stability}

Based on the data of CSG, this paper calculates the system critical clearance time under two load models: ZIP load and 40\% motor load 60\% constant resistance load in parallel. The result is showed in Table 4.

From Table 4 we can see that compared with ZIP load, the motor load will reduce the system critical clearance time. That demonstrates the viewpoint of Section 3.2: the motor load will consume more reactive power in the duration of fault, which is bad for voltage recovering and do harm to the system transient stability.

\section{Conclusions}

This paper analyzes the Mechanism and factors of commutation failure, studies the load models' impact on com- 
Table 3. Comparision of the sensitivity of each HVDC to AC system fault.

\begin{tabular}{ccccc}
\hline HVDC & ZIP Load & $40 \%$ Motor load & $50 \%$ Motor load & $60 \%$ Motor load \\
\hline Xin-an & 41 & 24 & 21 & 18 \\
Gao-zhao & 58 & 32 & 29 & 27 \\
Tian-guang & 69 & 21 & 19 & 18 \\
Jiangcheng & 59 & 34 & 30 & 28 \\
Yung-guang & 64 & 24 & 23 & 21 \\
Nuozhadu & 49 & 29 & 29 & 29 \\
Xiluodu & 69 & 27 & 27 & 27 \\
\hline
\end{tabular}

Table 4. Comparision of system critical clearance time under different load models.

\begin{tabular}{ccc}
\hline \multirow{2}{*}{ Fault Node } & \multicolumn{2}{c}{ System Critical Clearance Time (s) } \\
\cline { 2 - 3 } & ZIP Load & $40 \%$ Motor Load \\
\hline Suidong & 0.34 & 0.26 \\
Zhaoqing & 0.66 & 0.54 \\
Baoan & 0.28 & 0.22 \\
Beijiao & 0.34 & 0.28 \\
Echeng & $>1.5$ & $>1.5$ \\
\hline
\end{tabular}

mutation failure based on simulation. The conclusions of this paper are:

1) As the motor load can provide inertia to the system at the instance of fault, it is better than ZIP load in preventing voltage from declining, reducing the possibility of commutation failure and reducing the fault region that may lead to commutation failure occur in more than one inverters.

2) In the duration of fault, the motor load will enlarge the lack of reactive power and damage the system transient stability.

The conclusion of this paper would be useful in simulation and engineering applications.

\section{References}

[1] Zhao, W.J. (2004) Engineering Technology of HVDC Transmission System. China Electric Power Press, Beijing, 125.

[2] Yang, W.D., Xu, Z. and Han, Z.X. (2000) Special Issues and Suggestions on Multi-Infeed AC/DC Power Systems. Power System Technology, 24, 13-17.

[3] Xu, Z. (1998) AC/DC and DC/DC Interactions of Multiple HVDC Links Terminating in the Same AC System. Power System Technology, 22, 16-19.

[4] Thio C. V., Davise J. B. and Kent K. L. (1996) Commutation Failures in HVDC Transmission Systems. IEEE Transactions on Power Delivery, 11, 946-957. http://dx.doi.org/10.1109/61.489356

[5] Lin, L.X., Zhang, Y., Zhong, Q. and Zong, X.H. (2006) A Survey on Commutation Failures in Multi-infeed HVDC Transmission Systems. Power System Technology, 30, 40-46.

[6] Chen, S.Y., Li, X.N., Yu, J., Li, T., Lv, P.F. and Yin, Y.H. (2005) A Method Based on the Sin-Cos Components Detection Mitigates Commutation Failure in HVDC. Proceedings of the CSEE, 25, 1-6.

[7] Ou, K.J., Ren, Z. and Jing, Y. (2003) Research on Commutation Failure in HVDC Transmission System Part 1: Commutation Failure Factors Analysis. Electric Power Automation Equipment, 23, 5-8.

[8] Hao, Y.D. and Ni, R.B. (2006) Analysis on Influence Factors of Commutation Failure in HVDC. High Voltage Engineering, 32, 38-41.

[9] Hong, C. (2011) Simulation Analysis on the Commutation Failure and Power Recovery Characteristic of an Actual DC Transmission System. Southern Power System Technology, 5, 1-7.

[10] Rahimi, E., Gole, A.M., Davies, J.B., Fernando, I.T. and Kent, K.L. (2006) Commutation Failure in Single- and Multi-infeed HVDC Systems. The 8th IEE International Conference, London, 182-186.

[11] Rahimi, E., Gole, A.M., Davies, J.B., Fernando, I.T. and Kent, K.L. (2011) Commutation Failure Analysis in Multi- 
Infeed HVDC Systems. IEEE Transactions on Power Delivery, 26, 378-384. http://dx.doi.org/10.1109/TPWRD.2010.2081692

[12] Power Research Institute of CSG (2012) Research on AC/DC Interaction and Solution in China Southern Power Grid Receiving End System. Research Report of CSG.

[13] (1997) Guide for Planning DC Links Terminating at AC Locations Having Low Short Circuit Capacities. IEEE Std 1204, 5.

[14] Li, X.Y. (1998) Operation and Control of HVDC Transmission System. Science Press, Beijing, 2-4.

[15] Kundur, P. (1994) Power System Stability and Control. McGraw-Hill, New York.

[16] Wang, Z.F., Xue, Y.S. and Yang, W.D. (2006) Influences of Load Models on DC/AC System Stability. Automation of Electric Power Systems, 30, 13-16.

[17] He, Y.Z. and Wen, Z.Y. (2001) Power System Analysis. Huazhong University of Science and Technology Press, Wuhan, 77-78.

[18] Xu, Z.X. (2006) Dynamic Load Modeling for Transient Stability Study of Regional Interconnected Power System. Electric Power, 38, 15-19.

[19] Zhang, P.F., Luo, C.L., Meng, Y.J. (2006) Impact of Dynamic Electric Load Model Proportion on Power System Stability. Relay, 34, 24-26.

[20] Liao, M.C., Cai, G.L. and Zhang, Y.J. (2009) Transient Voltage Stability of Received Power Grid in AC/DC Hybrid Power Systems. Power System Protection and Control, 37, 5-8. 